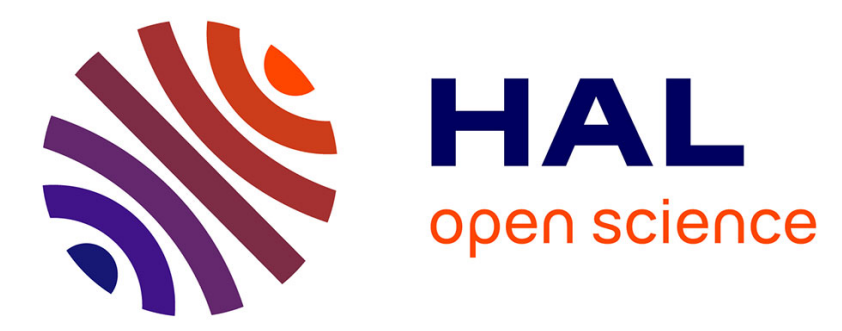

\title{
Development of a bio-inspired angular acceleration sensor : towards the non-invasive investigation of inner ear pathologies
}

Etienne Puyoo, Laurent Gremillard, Alice Vieren, Eugen Ionescu, Lucian Roiban

\section{To cite this version:}

Etienne Puyoo, Laurent Gremillard, Alice Vieren, Eugen Ionescu, Lucian Roiban. Development of a bio-inspired angular acceleration sensor : towards the non-invasive investigation of inner ear pathologies. 2021 Symposium on Design, Test, Integration \& Packaging of MEMS and MOEMS (DTIP), Aug 2021, Paris (virtuel), France. pp.1-4, 10.1109/DTIP54218.2021.9568668 . hal-03411900

\author{
HAL Id: hal-03411900 \\ https://hal.science/hal-03411900
}

Submitted on 2 Nov 2021

HAL is a multi-disciplinary open access archive for the deposit and dissemination of scientific research documents, whether they are published or not. The documents may come from teaching and research institutions in France or abroad, or from public or private research centers.
L'archive ouverte pluridisciplinaire $\mathbf{H A L}$, est destinée au dépôt et à la diffusion de documents scientifiques de niveau recherche, publiés ou non, émanant des établissements d'enseignement et de recherche français ou étrangers, des laboratoires publics ou privés. 


\section{Development of a bio-inspired angular acceleration sensor : towards the non-invasive investigation of inner ear pathologies}

\author{
Etienne Puyoo \\ Institut des Nanotechnologies de Lyon \\ INSA de Lyon \\ Villeurbanne, France \\ etienne.puyoo@insa-lyon.fr \\ Laurent Grémillard \\ Matériaux : Ingénierie et Science \\ INSA de Lyon \\ Villeurbanne, France \\ laurent.gremillard@insa-lyon.fr
}

\author{
Alice Vieren \\ Matériaux: Ingénierie et Science \\ INSA de Lyon \\ Villeurbanne, France \\ alice.vieren@insa-lyon.fr \\ Eugen Ionescu \\ Institut de l'Audition \\ Institut Pasteur, INSERM unité 1120 \\ Lyon, France \\ eugen.ionescu@chu-lyon.fr
}

\author{
Lucian Roiban \\ Matériaux : Ingénierie et Science \\ INSA de Lyon \\ Villeurbanne, France \\ ioan-lucian.roiban@insa-lyon.fr
}

\begin{abstract}
In this paper, we introduce the development of a bio-inspired system that mimics the angular acceleration sensor function provided by the vestibular system. The prototype is made of a Plexiglas piece that presents the pattern of a semicircular canal at scale 10, with a simplified geometry. A flexible piezoresistive cantilever integrated on polyimide substrate is used to model the electro-mechanical transduction of the cupula/hair cells system. The electro-mechanical response of the complete system is analyzed when submitted to both pulse and sine rotational excitations. It is demonstrated that the biomimetic system only responds to one rotational axis, and is also sensitive to the rotational direction. We also validate that the prototype actually responds to the expected medium vestibular frequencies (from $0.16 \mathrm{~Hz}$ to $0.64 \mathrm{~Hz}$ ).
\end{abstract}

Keywords-bio-inspired angular acceleration sensor, vestibular system, inner ear, flexible sensor, piezoresistive cantilever.

\section{INTRODUCTION}

The inner ear is a membranous sensory organ that provides auditory and balance functions [1]. Its posterior part, the vestibular system, is sensitive to low frequency vibrations (from $0.05 \mathrm{~Hz}$ to $10 \mathrm{~Hz}$ ) specific to the spectrum of body movements [2]. The vestibular system contains three semicircular canals perpendicular to each other that can monitor rotational head motions through a cascade of biomechanical events [3]. Under rotation with respect to the symmetry axis of the semicircular duct, the displacement of the endolymph fluid deflects the cupula and activates vestibular hair cells that are directly connected to the vestibular contingent of the eighth cranial nerve. The semicircular duct system is especially responsible for the horizontal vestibulo-ocular reflex that helps to stabilize head and gaze motions and is essential for the perception and control of movement.

Today, it is estimated that about $20 \%$ to $30 \%$ of the global population suffers from balance disorders, due to some defects of the vestibular system in $85 \%$ of cases [1]. Because of the fragility, microscopic dimensions and complexity of the inner ear structures, relatively few steps have been taken to date in the specific field of treatments dedicated to the inner ear diseases. In this context, our aim is to develop an artificial vestibular system [4-6] that thoroughly represents the physiology of the inner ear in order to sustain or confirm new pathophysiological mechanism theories and to propose new treatment procedures [7-9].

Several numerical model that describe the fluidics of the semicircular canal, the fluid/cupula interaction and the kinocillia mechanics have already been proposed in literature [10-13]. However, these models were never compared to a physical model and were not applied to solve and treat any pathology. Concerning the physical models, few biomimetic gyroscopes were presented in literature to develop implants but with morphologies that differ from the real physiology of the vestibular system [4-6].

Among its potential impact in clinical medicine, we also believe that the development of a physiologically realistic physical model could be used as an educational tool for academics and hospital practitioners to illustrate different physiological processes as, for example, the vestibulo-ocular reflex. Before to reach these two long-term ambitious goals, the first step is to validate the bio-inspired angular acceleration sensor function provided by the semicircular duct system in a simplified geometrical configuration. Here, the fabrication process of the biomimetic system is first presented. Its electromechanical response to both pulse and sine rotational excitations is then analyzed and validated.

\section{INTEGRATION OF THE ANGULAR ACCELERATION SENSOR}

As shown in figure 1a, the prototype is made of a Plexiglas piece that presents the pattern of a semi-circular canal at scale 10 . It represents an ideal case because the dimensions are perfectly controlled, and because the stiffness of the material prevents from any outside interaction. The piece has been designed in two symmetrical parts which are stuck and made airtight, so it can be filled with a fluid to model the endolymph. We have also provided a lateral slot to insert a piezoresistive sensor and drilled two holes on the top to inject the fluid within the mock-up. The circular canal, 
a)

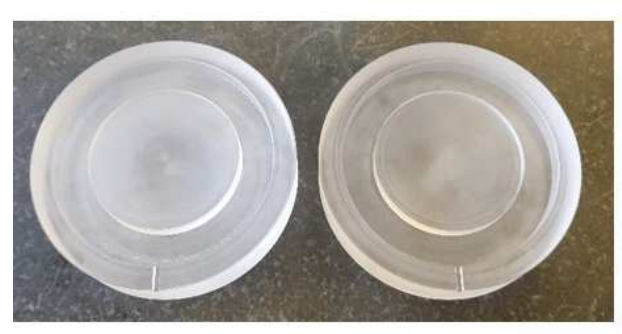

b)

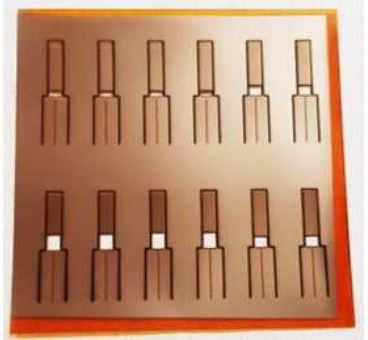

c)

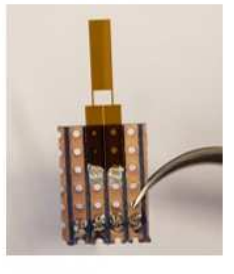

d)

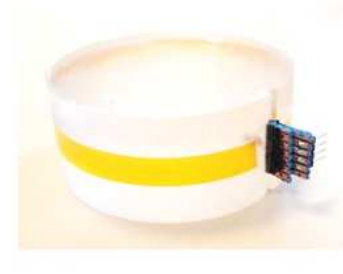

Figure 1: a) Machined Plexiglas part; flexible piezoresistive cantilevers b) after fabrication and c) mounted on PCB; d) photograph of the assembled prototype.

lateral slot and holes were all patterned by milling within the Plexiglas blocks. Note that the canal has a square section of $1 \mathrm{~cm}^{2}$ and a radius $r$ of $2.75 \mathrm{~cm}$.

To model the electro-mechanical transduction of the cupula/hair cells system, we have used a flexible piezoresistive cantilever connected to a printed-circuit-board (figure $1 \mathrm{~b}$ and 1c). This cantilever was inserted within the slot of the Plexiglas mock-up and then stuck with a silicone paste. After complete drying of the silicone paste (24h hour at room temperature), the circular canal was finally filled with deionized water and sealed with an adhesive tape (photograph of the assembled prototype provided in figure 1d).

Concerning the elaboration process of the piezoresistive cantilever, we have implemented the two steps process depicted in figure 2. First, a metallic thin film is grown on polymer substrate by e-beam evaporation. Then, partial and/or total UV LASER cutting is used to define the cantilever and piezoresistive element geometries. For this particular study, we have deposited a $10 \mathrm{~nm}$ thick Pd film on a $75 \mu \mathrm{m}$ thick polyimide substrate. To pattern the flexible transducer, a UV Laser spot (wavelength of $355 \mathrm{~nm}$, output power of $0.8 \mathrm{~W}$, spot size of $6.25 \times 10^{-6} \mathrm{~cm}^{2}$, pulse duration of $10 \mathrm{~ns}$ and repetition rate of $15 \mathrm{kHz}$ ) was scanned over the surface at a speed of 80 $\mathrm{mm} \cdot \mathrm{s}^{-1}$. Eighteen LASER passes were applied for a complete cutting of the polyimide film (to pattern the cantilever geometry) while only three subsequent passes were used to pattern the two metallic pads on the base of the cantilever (these pads are used for electrical connection to the PCB). As shown in figure $1 \mathrm{c}$, the cantilever has a total length of $12.5 \mathrm{~mm}$. It integrates two $1.5 \mathrm{~mm}$ long legs at its base that both have a width of $300 \mu \mathrm{m}$. Each of these two legs integrates Pd piezoresistive elements arranged in series with a total resistance $R_{0}$ of $1.91 \mathrm{k} \Omega$.

1. e-beam evaporation of a metallic thin film on polyimide substrate.

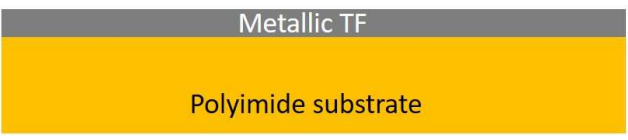

\section{UV LASER cutting (partial of total)}

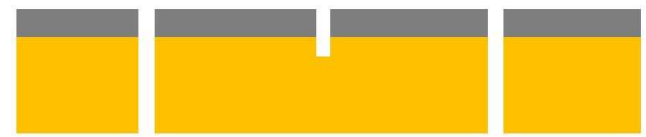

Figure 2: Illustration of the two steps process used to develop piezoresistive cantilevers.

\section{ELECTRO-MECHANICAL RESPONSE ANALYSIS}

The sensitivity to deformation of the flexible cantilever was characterized before its integration within the Plexiglas mock-up. A cantilever deflection method was performed according to the experimental procedure described in references $[14,15]$. The $12.5 \mathrm{~mm}$ long cantilever mounted on PCB was clamped in a vice while a variable deflection was applied at its extremity by a motorized actuator. A fixed bias of $0.1 \mathrm{~V}$ was applied to the device while the current was measured at the same time for different deflection levels ranging from $0 \mu \mathrm{m}$ to $500 \mu \mathrm{m}$. Both tensile and compressive strains were applied to the $\mathrm{Pd}$ piezoresistive elements by placing the Pd metallized face of the cantilever up and down, respectively. The relative change in resistance of the cantilever is plotted as a function of the deflection level in figure 3. From the two characteristics carried out under tensile and compressive strains, we observe a good linearity on the whole deflection range and extract a sensitivity coefficient $\alpha$ of $2.54 \mathrm{~m}^{-1}$.

The electro-mechanical response of the complete prototype was then analyzed under pulse rotational excitations. As shown in figure $4 \mathrm{a}$, the mock-up was initially set horizontally and then rotated by hand with respect to the three space axis. Under rotation with respect to the symmetry axis of the canal, the cantilever is allowed to bend as a result of the relative motion between the fluid and the canal. This bending leads to a resistance variation that can be monitored to probe rotational motion. The resistance of the cantilever is plotted in figure $4 \mathrm{~b}$ as a function of time for different pulse excitations around the three axis. From this graph, we can

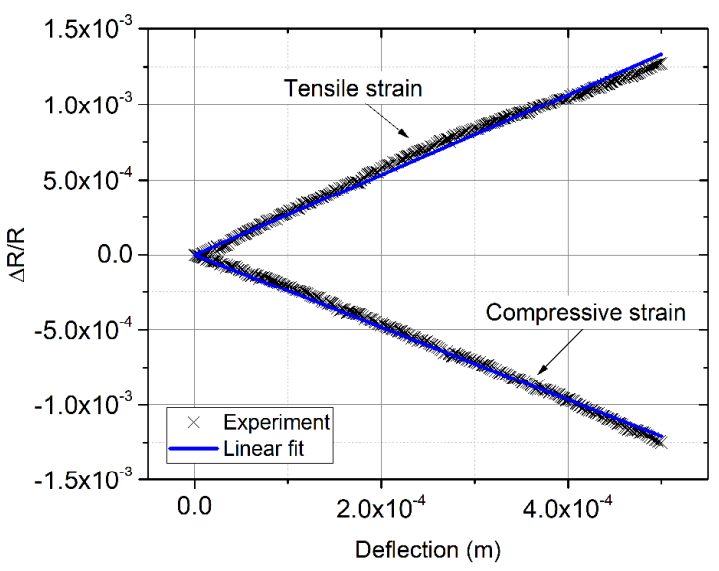

Figure 3: Sensitivity to deformation of the flexible piezoresistive cantilever in a bending configuration. 


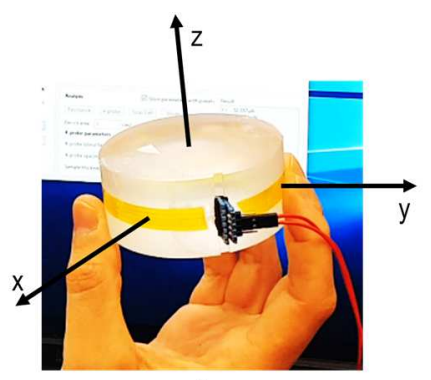

a)

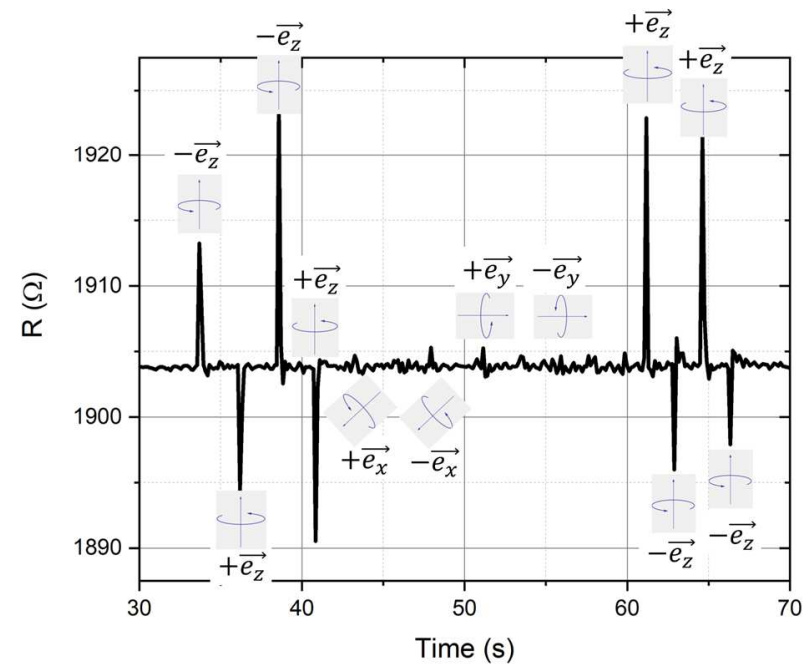

b)

Figure 4: a) Orientation in space of the bio-inspired system and b) response of the system to pulse rotational excitations.

conclude that the biomimetic system only responds to one rotational axis (the vertical $z$ axis), and is also sensitive to the rotational direction. As expected, a resistance decrease is observed for a positive rotation around the z-axis for which the Pd piezoresistive elements are submitted to compressive strain while the cantilever resistance obviously increases in the opposite direction under tensile strain.

Concerning the response to sine rotational excitation, the physical model was fixed on a swivel chair used to diagnose vestibular diseases (figure 5a), and rotated at different oscillation frequencies. The graph in figure $5 \mathrm{~b}$ compares the angular position $\theta$ of the swivel chair over time to the resistance of the piezoresistive cantilever. From this experiment that we have performed at three different excitation frequencies from $0.16 \mathrm{~Hz}$ to $0.64 \mathrm{~Hz}$ (i.e. angular frequencies from $1.0 \mathrm{rad} . \mathrm{s}^{-1}$ to $4.0 \mathrm{rad} . \mathrm{s}^{-1}$ ), we could validate that the prototype actually responds to the expected medium vestibular frequencies.

To deeper analyze the response of the system under forced oscillations, we have readapted the analytical model developed by Steinhausen in 1933 [16] that describes the dynamics of the semicircular canal as a strongly damped torsional pendulum. According to [16], the displacement $x$ of the fluid over time at the center of the canal is indeed governed by the following equation:

$$
m \frac{d^{2} x}{d t^{2}}+\lambda\left(\frac{d x}{d t}-\frac{d \xi}{d t}\right)+k(x-\xi)=0
$$

Where $\xi$ is the canal wall displacement, $m$ the mass of the fluid, $\lambda$ the friction coefficient governed by the viscous drag
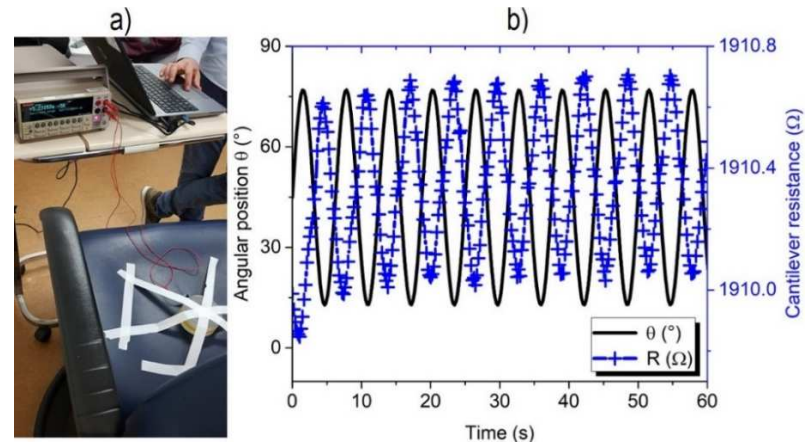

Figure 5: a) Prototype fixed on swivel chair and b) response to sine rotational excitation.

of the fluid inside the canal and $k$ the cupula stiffness [16]. In permanent regime under forced oscillations at the angular frequency $\Omega$, the deflection $D(t)$ of the cupula (i.e. of the cantilever) is assumed to be equal to the difference between the fluid displacement $x(t)$ and the wall displacement $\xi(t)$ :

$$
D(t)=x(t)-\xi(t)=x(t)-r \theta_{0} \cos (\Omega t)
$$

Where $r$ is the canal radius and $\theta_{0}$ the oscillation amplitude imposed by the swivel chair. Equation (1) can be then rewritten as a function of the variable $D(t)$ :

$$
\frac{d^{2} D}{d t^{2}}+2 \delta \frac{d D}{d t}+\omega_{0}^{2} D=\Omega^{2} r \theta_{0} \cos (\Omega t)
$$

Where $\delta=\frac{\lambda}{2 m}$ is the damping coefficient of the system and $\omega_{0}=\sqrt{\frac{k}{m}}$ its eigenfrequency.

In permanent regime, the cantilever deflection $D(t)$ is supposed to have the same mathematical form as the second member of equation (3) and is expressed as:

$$
D(t)=D_{m} \cos (\Omega t+\phi)
$$

Where $D_{m}$ and $\phi$ are respectively the cantilever deflection amplitude and phase. By solving equation (3) in complex formalism, the deflection amplitude and phase are found to be expressed as:

$$
\begin{aligned}
& D_{m}=\frac{r \theta_{0} \Omega^{2}}{\sqrt{\left(\omega_{0}^{2}-\Omega^{2}\right)^{2}+(2 \delta \Omega)^{2}}} \\
& \phi=-\arctan \left(\frac{2 \delta \Omega}{\omega_{0}^{2}-\Omega^{2}}\right)
\end{aligned}
$$

\begin{tabular}{|c|c|c|c|c|c|c|}
\hline \multicolumn{5}{|c|}{ Fixed parameters } & \multicolumn{2}{|c|}{$\begin{array}{c}\text { Extracted } \\
\text { parameters }\end{array}$} \\
\hline $\begin{array}{l}R_{0} \\
(\Omega)\end{array}$ & $\begin{array}{c}\alpha \\
\left(\mathrm{m}^{-1}\right)\end{array}$ & $\begin{array}{c}r \\
(\mathrm{~cm})\end{array}$ & $\begin{array}{c}\Omega \\
\left(\operatorname{rad} . \mathrm{s}^{-1}\right)\end{array}$ & $\begin{array}{l}\theta_{0} \\
\left({ }^{\circ}\right)\end{array}$ & $\begin{array}{c}\omega_{0} \\
\left(\mathrm{rad} . \mathrm{s}^{-1}\right)\end{array}$ & $\begin{array}{c}\delta \\
\left(\mathrm{s}^{-1}\right)\end{array}$ \\
\hline 1910.4 & \multirow{3}{*}{2.54} & \multirow{3}{*}{2.75} & 1.0 & 33.6 & 4.8 & 138 \\
\hline 1910.6 & & & 2.0 & 28.0 & 2.9 & 220 \\
\hline 1910.5 & & & 4.0 & 11.2 & 3.9 & 197 \\
\hline
\end{tabular}

Finally, the resistance $R(t)$ of the cantilever is given by:

$$
R(t)=R_{0}(1+\alpha D(t))
$$

Where $R_{0}$ is the cantilever resistance at rest and $\alpha$ the cantilever sensitivity coefficient extracted from figure 3 .

Table 1: Parameters used in the model. 
a)

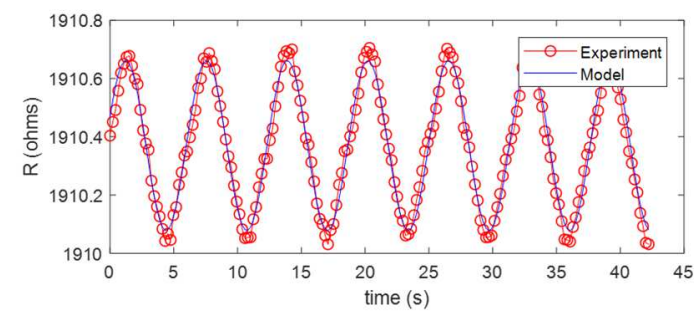

b)

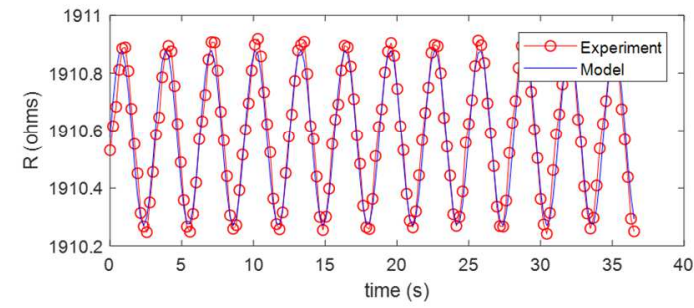

c)

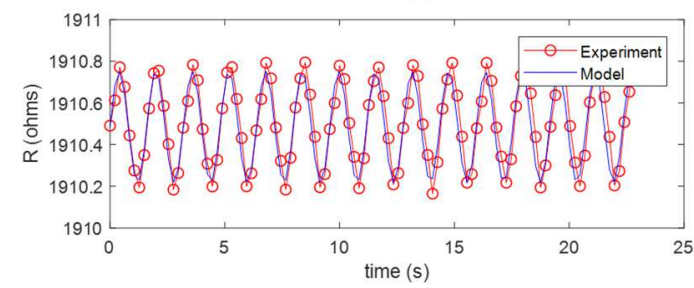

Figure 6: Comparison of the model with the experimental data for an imposed angular frequency of a) $1.0 \mathrm{rad}^{-1} \mathrm{~s}^{-1} \mathrm{~b}$ ) $2.0 \mathrm{rad} . \mathrm{s}^{-1}$ and c) $4 \mathrm{rad} . \mathrm{s}^{-1}$.

All the known parameters $\left(R_{0}, \alpha, r, \Omega, \theta_{0}\right)$ were fixed in the model while the damping coefficient $\delta$ and eigenfrequency $\omega_{0}$ of the system were set as free parameters (see Table 1). It should be noted that three slightly different $R_{0}$ values were set in the model for the three tested angular frequencies. These slight resistance variations could be attributed to temperature fluctuations occurring during the experiment.

Equation (7) was finally used to fit the experimental data presented in figure 6. A good agreement between the model and the experiment is observed for the three imposed angular frequencies in permanent regime. From figure 6 , we extract an eigenfrequency $\omega_{0}$ in the range of $2.9-4.8 \mathrm{rad}^{-1} \mathrm{~s}^{-1}$ and a damping coefficient $\delta$ of $138-220 \mathrm{~s}^{-1}$. These two extracted parameters can be used to estimate two characteristic parameters of our simplified physical model: the cupula time constant $T_{C}$ and the viscous time scale $T_{V}$. The first one also called the slow time scale [17] is inversely proportional to the stiffness of the cupula as given by: $T_{C}=\frac{\lambda}{k}=\frac{2 \delta}{\omega_{0}^{2}}$. According to [17], the viscous time scale relates the fluid inertia to its viscous force: $T_{V}=\frac{m}{\lambda}=\frac{1}{2 \delta}$. From the extracted $\omega_{0}$ et $\delta$ values, we estimate a cupula time constant $T_{C}$ in the range of $12 \mathrm{~s}-52 \mathrm{~s}$ and a viscous time scale $T_{V}$ in the range of $2.5 \times 10^{-3} \mathrm{~s}$. These estimations agree with data obtained on real organs as reported in reference [17] which confirms that our bioinspired system properly responds to the expected vestibular frequencies.

\section{CONCLUSION}

In summary, we have developed a bio-inspired angular acceleration sensor that mimics the function of a semicircular canal. This physical model was made from two symmetrical micro-machined Plexiglas pieces that integrate a transduction system based on a flexible piezoresistive cantilever. Under excitation around the three space axis, we could demonstrate that the system only responds to one rotational axis and is also sensitive to the rotational direction. Finally, the response analysis of the system under forced oscillations enables us to validate that our prototype responds to the expected medium vestibular frequencies.

\section{ACKNOWLEDGMENT}

The authors would like to thank Joëlle Grégoire for her technical support on the NanoLyon technological platform.

\section{REFERENCES}

[1] C. F. Santos, J. Belinha, F. Gentil, M. Parente and R. N. Jorge, "An alternative 3D numerical method to study the biomechanical behaviour of the human inner ear semicircular canal", Acta of Bioengineering and biomechanics, vol. 19(1), pp. 3-15, 2017.

[2] L. Sakka and E. Vitte, "Anatomie et physiologie du système vestibulaire, Morphologie", vol. 88, pp. 117-126, 2004 (DOI: 10.1016/S1286-0115(04)98134-9).

[3] R. David, A. Stoessel, A. Berthoz, F. Spoor and D. Bennequin, "Assessing morphology and function of the semicircular duct system: introducing new in-situ visualization and software toolbox", Scientific Reports, vol. 6, pp. 32772, 2016.

[4] C.M. Andreou, Y. Pahitas and J. Georgiou, "Bio-inspired micro-fluidic angular-rate sensor for vestibular prostheses", Sensors, vol. 14, pp. 13173-13185, 2014

[5] D. Obrist, S. Hegemann, D. Kronenberg, O. Häuselmann and T. Rösgen, "In vitro model of a semicircular canal: design and validation of the model and its use for the study of canalithiasis", Journal of Biomechanics, vol. 43(6), pp. 1208-1214, 2010.

[6] J. van Tiem, J. Groenesteijn, R. Sanders and G. Krijnen, "3D printed bio-inspired angular acceleration sensor," 2015 IEEE SENSORS, 2015, pp. 1-4, doi: 10.1109/ICSENS.2015.7370543.

[7] E.C. Ionescu, N. Altami, A. Neagu, A. Ltaief-Boudrigua, S. Gallego, R. Hermann, E. Truy and H. Tai-Van, "Superior semicircular canal ampullae dehiscence as part of the third window abnormalities: a case study", Frontiers in Neurology, vol. 8, pp. 683, 2017.

[8] E.C. Ionescu, A. Coudert, P. Reynard, E. Truy, H. Tai-Van, A. LtaiefBoudrigua and F. Turjman, "Stenting the superior petrosal sinus in a patient with symptomatic superior semicircular canal dehiscence", Frontiers in Neurology, vol. 9, pp. 689, 2018.

[9] E.C. Ionescu, P. Reynard, A. Coudert, L. Roiban, A. Ltaief-Boudrigua and H. Tai-Van, "Superior semicircular canal dehiscence by superior petrosal sinus: proposal for classification", The Journal of International Advanced Otology, vol. 17, pp. 35, 2021.

[10] M. Muller, "Semicircular duct dimensions and sensitivity of the vertebrate vesitibular system", J. theor. Biol., vol. 167, pp. 239-256, 1994.

[11] R.K. Duncan and J.W. Grant, "A finite-element model of inner ear hair bundle micromechanics", Hearing Research, vol. 104, pp. 15-26, 1997.

[12] P. Selva, "Modélisation du système vestibulaire et modèles nonlinéaires de perception de l'orientation spatiale", $\mathrm{PhD}$ thesis, ISAE SUPAERO, 2009.

[13] P. Selva, J. Morlier and Y. Gourinat, "Toward a three-dimensional finite-element model of the human inner ear angular accelerometers sensors", International Journal for Computational Vision and Biomechanics (IJCV \& B), vol. 3 (2), pp. 149-156, 2010.

[14] R. Rafael, E. Puyoo and C. Malhaire, "Piezo-tunnel effect in $\mathrm{Al} / \mathrm{Al}_{2} \mathrm{O}_{3} / \mathrm{Al}$ junctions elaborated by atomic layer deposition", Journal of Applied Physics, vol. 112, pp. 184501, 2017.

[15] R. Rafael, E. Puyoo and C. Malhaire, "Piezo-tunneling strain sensors integrated on plastic by combining vacuum thin film coatings and $3 \mathrm{D}$ printing technologies”, Microsystem Technologies, vol. 26(12), pp. 3623-3628, 2020 .

[16] W. Steinhausen, "Über die Beobachtung der Cupula in den Bogengangsampullen des Labyrinths des lebenden Hechts", Pflügers Archiv für die gesamte Physiologie des Menschen und der Tiere, vol. 232, 500-512, 1933.

[17] D. Obrist, "Fluid mechanics of the inner ear", Habilitation Thesis, ETH Zürich, 2011, doi: 10.3929/ethz-a-007318979 